\title{
Efecto del uso de un sistema de información en el proceso contable de una empresa agroindustrial del distrito de San Hilarión
}

Effect of the use of an information system in the accounting process of an agro-industrial company of the San Hilarion district

Efeito da utilização de um sistema de informação no processo contábil de uma empresa agroindustrial do distrito de San Hilarion

Chujutalli Mendoza, Katia del Pilar
dchujutallime1 @ ucvvirtual.edu.pe
Universidad César Vallejo - Perú
ORCID: 0000-0001-7236-7993

Dávila Flores, Jorge

ddavilfl@ucvvirtual.edu.pe

Universidad César Vallejo - Perú ORCID: 0000-0002-9325-0572

Soto Abanto, Segundo Eloy

ssotoa@ucv.edu.pe

Universidad César Vallejo - Perú ORCID: 0000-0003-1004-5520

Villafuerte de la Cruz Avelino Sebastián avillafuerte@ucv.edu.pe

Universidad César Vallejo - Perú ORCID 0000-0002-9447-8683

\section{RESUMEN}

La presente investigación, tiene por objetivo determinar el efecto del uso de un sistema de información en el proceso contable de una empresa agroindustrial del distrito de San Hilarión, para esto se consideró una investigación de tipo aplicada, de diseño no experimental, de corte transversal y de alcance descriptivo, con una población de estudio una empresa del rubro agroindustrial y una muestra de los seis periodos evaluados 2015 al 2020. Para la recolección de datos se aplicó la entrevista y el análisis documental, se obtuvo como resultado que el uso del sistema de información tiene un efecto positivo en la empresa agroindustrial del distrito de San Hilarion, teniendo mejoras, reduciendo el tiempo de presentación de los estados financieros. Como conclusión se obtuvo que el uso del sistema de información ayuda y facilita tener de manera resumida y oportuna información útil para tomar decisiones.

Palabras claves: Sistema de información, sistemas, contable, empresa, contabilidad.

\begin{abstract}
The present research aims to determine the effect of the use of an information system in the accounting process of an agro-industrial company in the district of San Hilarión, for this, applied research was considered, of non-experimental design, of cross-sectional and descriptive scope, with a study population, a company in the agro-industrial sector and a sample of the six periods evaluated from 2015 to 2020. For data collection, the interview and documentary analysis were applied, the result was that the use of the Information has a positive effect on the agro-industrial company of the San Hilarion district, having improvements, reducing the time of presentation of the financial statements. As a conclusion, it was obtained that the use of the information system helps and facilitates having, in a summarized and timely manner, useful information for making decisions.
\end{abstract}

Keywords: Information system, systems, accountant, company, accounting.

\section{RESUMO}

O objetivo desta pesquisa é determinar o efeito da utilização de um sistema de informação no processo contábil de uma empresa agroindustrial do distrito de San Hilarión, para isso foi considerada uma pesquisa aplicada, delineamento não experimental, transversal e de escopo descritivo, com uma população de estudo, uma empresa do setor agroindustrial e uma amostra dos seis períodos avaliados de 2015 a 2020. Para a coleta de dados aplicou-se a entrevista e a análise documental, o resultado foi que a utilização da Informação tem um efeito positivo na empresa agroindustrial do distrito 
de San Hilarion, tendo melhorias, reduzindo o tempo de apresentação das demonstrações financeiras. Como conclusão, obteve-se que a utilização do sistema de informação auxilia e facilita ter, de forma resumida e tempestiva, informações úteis para a tomada de decisões.

Palavras-chave: Sistema de Informações, Systems, contador, companhia, contabilidade.

\section{INTRODUCCIÓN}

El comercio, la industria y los servicios se ven influenciados por el uso de sistema de información, al proporcionar una serie de herramientas que facilitan los procesos en las diferentes áreas de la empresa, como la contabilidad, las finanzas, la administración y la gestión (FuentesDoria et al., 2019). El problema de la investigación se da en la empresa debido a que no contaba con un sistema de información, esto venia ocasionando deficiencias en el proceso contable, presentando los informes financieros de manera errada y ha generado incumplimiento de los objetivos de la empresa. Se plantea el problema general: ¿cuál es el efecto del uso de un sistema de información en el proceso contable de una empresa agroindustrial del distrito de San Hilarión?, de manera específica: ¿cómo analizar el uso de sistemas de información dentro de una empresa agroindustrial del distrito de San Hilarión?, ¿cómo se realiza el proceso contable dentro de una empresa agroindustrial del distrito de San Hilarión? Se presentó como objetivo general: determinar el efecto del uso de un sistema de información en el proceso contable de una empresa agroindustrial del distrito de San Hilarión; y de manera específica se planteó los siguientes objetivos: analizar el uso de un sistema de información dentro de una empresa agroindustrial del distrito de San Hilarión, identificar el estado del proceso contable dentro de una empresa agroindustrial del distrito de San Hilarión. La investigación se justificó por su relevancia social, por su valor de utilidad metodológica, e implicaciones prácticas, y se planteó la siguiente Hipótesis: el efecto del uso de un sistema de información en el proceso contable de una empresa agroindustrial fue positivo.

\section{FUNDAMENTO TEÓRICO}

\subsection{Antecedentes}

En la investigación "Determinantes de la utilidad percibida y del sistema de información contable del usuario final en las PYME" Realizada por de (Andarwati et al., 2020) fue de tipo básica y diseño no experimental, consideraron como objetivo analizar la información vertida por el sistema contable para utilidad en la toma de decisiones financiera de las pymes, han tomado en cuenta una población de 183 empresarios fueron encuestados, la información se ha recolectado y concluyeron que, los sistemas de información contable desempeñan un papel importante para las pymes minoristas. Los datos de esta investigación muestran los efectos de la facilidad de uso percibida, la calidad del sistema y la información sobre la satisfacción del usuario final de la información contable a través de la percepción de la utilidad de las pequeñas y medianas empresas.

Por otro lado, en la investigación "Proceso contable: herramienta financiera para la toma de decisiones en las PYMES Parroquia El Esfuerzo" de (Rodríguez et al., 2019) dirigido a 42 microempresas del sector, consideró el objetivo determinar el grado de influencia de los procesos contables como herramienta financiera en la toma de decisiones. El estudio se basa en la observación documental, se aplicó el método inductivo-deductivo, de alcance descriptivo, explicativo, no experimental, transeccional. La medición se basó en la escala de Likert. Para analizar de los datos se utilizó el software SPSS V.22. En conclusión, el proceso contable como herramienta financiera se enfoca como fórmula para el desarrollo de las microempresas en el sector.

De acuerdo con la investigación "El impacto de los sistemas de información integrados en la contabilidad de gestión: El caso de Croacia” de (Pervan \& Dropulic', 2019) aplicada a 108 empresas croatas, tuvo el propósito de analizar los efectos de la implementación de un sistema integrado en los cambios de la contabilidad de gestión. El estudio es correlacional, descriptivo, 
explicativo, cuantitativo, no experimental, de corte transversal. Se elaboró un cuestionario y concluyeron que la implementación de un sistema realizó cambios en la contabilidad de gestión con mayores capacidades analíticas, reduce el tiempo de presentación de informes, aumenta la frecuencia de presentación de informes y aumenta el número de informes.

Como se indica en la investigación de Elizalde (2019) sobre "Gestión contable como base fundamental para las decisiones generales" cuyo objetivo es analizar la gestión contable como base fundamental para las decisiones gerenciales. Con el propósito de consumar con dicho objetivo se manejó una metodología de tipo documental con diseño bibliográfico, examinando los fundamentos teóricos sobre la gestión contable, etapas, toma de decisiones, tipos y proceso. Fue realizada la lectura e interpretación de los textos seleccionados para formular un análisis y generar unas conclusiones del contenido. Para la fase en la cual se consolida la información, se utiliza una base de datos elaborada a tal efecto. En el desarrollo de la etapa de análisis de los documentos y fuentes, se elaboraron las fichas de contenido textual, que permitieron tabular los datos obtenidos. Logrando establecer que la gestión contable es la base sobre la cual se fundamentan las decisiones gerenciales y, por tanto, las decisiones financieras. En consecuencia, no existe actividad económica ajena al registro y afectación de las técnicas de la ciencia contable.

Según Morales-Antamba et al. (2019) en su investigación "Importancia de los fundamentos contables. Aplicación práctica de un proceso contable en una empresa comercial" Como objetivo del trabajo se planteó elaborar el Estado de Resultados y el Estado de Situación Financiera de una empresa comercial, como productos finales de la aplicación del proceso contable. El proyecto estuvo encaminado en un tipo de investigación no experimental orientado a observar los acontecimientos que se suscitaron, más no a manipular el objeto de estudio, y; a su vez se consideró un diseño de investigación mixto (bibliográfico y campo). Entre los principales resultados se destacan que con la elaboración del balance general o estado de situación financiera se dio a conocer a la empresa el valor total del inventario final de sus mercaderías; el mismo que desconocían, además determinar el monto de sus deudas con terceros y del capital propio; por otro lado, a través del estado de resultados se estableció la ganancia real mensual que generan. Como principal conclusión se puede mencionar que si bien es cierto la normativa tributaria establece las condiciones para ser obligados o no a llevar contabilidad, esto no debería ser un limitante para que las empresas implementen un adecuado control de su información económica-financiera, que les permita a través de los estados financieros tomar las mejores decisiones para sus negocios.

En la investigación "Diagnóstico de los sistemas de información en las empresas priorizadas según los requerimientos actuales" de (Castillo \& Pérez, 2017) un estudio que tuvo como objetivo comprobar la etapa actual del sistema de información en las compañías prevalecidas por la Representación territorial del CITMA en Pinar del Río. Se trató de un estudio de tipo cualitativo, de investigación-acción, participativa, el estudio tuvo una muestra de estudio 25 empresas. Asimismo, se aplicó el método histórico-lógico, se aplicó el instrumento de cuestionario con la finalidad de recoger datos sobre el proceder de los sistemas de información en el perfeccionamiento y los procesos de gestión de la información. Concluyó que las empresas constatadas en el estudio requieren continuar actuando para elevar la eficiencia de los sistemas de información. Con la implementación de la norma internacional 27001 adoptada por Cuba, constituye una práctica muy necesaria para garantizar la competitividad de las organizaciones y demostrar la seguridad de la información a los clientes y demás partes interesadas.

Asimismo, según Crespo et al. (2020) en su investigación "Mejora Continua en el proceso contable y su aporte en la competitividad de las MIPYMES en la Provincia de El Oro (Ecuador)" su objetivo se centra en describir la mejora continua en los procesos contables para defender la competitividad en las MIPYMES en la Provincia de El Oro, tiene un alcance explicativo basado en el estudio de campo que permitió fijar los errores, es de alcance descriptivo y correlacional se aplica una metodología mixta (cualitativa y cuantitativa). la población de estudio fue de 1,406 
microempresas, solo se aplicó una muestra de 170 empresas MiPymes; para obtener los resultados se aplicó una encuesta.

Según Farida et al. (2021) en su investigación "Calidad y eficacia de los sistemas de información contable" Esta investigación se realizó mediante un cuestionario distribuido a 51 empleados que laboran en la Unidad de Contabilidad de Ministerios e Instituciones utilizando el método de muestreo intencional. Se utilizó el modelado de ecuaciones estructurales utilizando el programa Lisrel 8.80 para analizar los datos. Los resultados mostraron que la Implementación del Sistema de Información Contable tuvo un efecto positivo significativo en el Desempeño Organizacional a través de la Calidad de la Información Financiera.

\subsection{Teorías relacionadas (Variables, dimensiones)}

El uso del sistema de información está comprendido por la sistematización, procesamiento, evaluación, información e inversión para ser más eficientes y tienen el objetivo de llevar la contabilidad, es decir logran obtención de información financiera de las operaciones realizadas en un periodo (Elizalde, 2019) y comprende cinco dimensiones. La primera centrada en la sistematización, significa establecer un sistema de información financiera, el cual debe ser seleccionado, diseñado e instalado, por lo que, agrupa indicadores como el diseño de los procedimientos, el interfaz del sistema y el manual de sistematización. La segunda estará centrada en el procesamiento, consiste en la elaboración de los estados financieros resultantes de las transacciones realizadas por una entidad, por lo que, tiene como indicadores la captación de los datos financieros, la clasificación de los datos financieros, el registro de datos financieros y la elaboración de estados financieros. La tercera comprende la evaluación, pues califica el efecto de las transacciones celebradas por una entidad económica, respecto a ello, esta dimensión agrupa indicadores como el análisis de la información financiera, lo métodos de análisis y la responsabilidad de la decisión. La cuarta comprende la información, la cual incluye la comunicación de los resultados obtenidos durante la fase de evaluación financiera de la organización a través de un informe bien estructurado. Y la quinta comprende la inversión la cual incluye el costo de implementación del sistema, y los gastos incurridos para su mantenimiento (Elizalde, 2019).

El proceso contable se rige en una serie de procedimientos en la recopilación, procesamiento y comunicación de información financiera. Implica pasos específicos para registrar, clasificar, resumir e interpretar transacciones y eventos para una entidad (Morales-Antamba et al., 2019). Y se dimensiona en la clasificación de documentos, donde Torres (2019), afirmó que la clasificación es el procedimiento que consiste en aglomerar de forma diferenciada los documentos, conforme con los elementos de origen, secuencia original u otros elementos. El autor Patricio (2017), indicó que el registro de operaciones su finalidad es registrar los movimientos realizados por la compañía, para elaborar el balance de comprobación e integrar los estados financieros. Asimismo, Guzmán (2018), definió que el análisis de cuentas se ajusta con la información hecha por la compañía, y su objetivo es admitir el control, la rendición de cuentas a los propietarios, inversores o accionistas y medir eficientemente los resultados.

\section{PROCEDIMIENTOS METODOLÓGICOS}

\subsection{Tipo y diseño de la investigación}

La investigación es de tipo aplicada, porque se considera teorías de diferentes autores, es de diseño no experimental por que se recolectará la información tal como está; no se manipulará ninguna variable, y de corte transversal por que estará comprendido en tiempo determinado. 


\subsection{Operacionalización de variables}

\section{Tabla 1}

Uso del sistema de información

\begin{tabular}{|c|c|c|c|c|c|}
\hline Variable & Definición conceptual & Definición operacional & Dimensiones & Indicadores & $\begin{array}{l}\text { Escala de } \\
\text { medición }\end{array}$ \\
\hline \multirow{14}{*}{$\begin{array}{l}\text { Uso de } \\
\text { sistema de } \\
\text { información }\end{array}$} & \multirow{14}{*}{$\begin{array}{l}\text { El uso del sistema de } \\
\text { información } \\
\text { comprendido por la } \\
\text { sistematización, } \\
\text { procesamiento, } \\
\text { evaluación, información e } \\
\text { inversión para ser más } \\
\text { eficientes y tienen el } \\
\text { objetivo de llevar la } \\
\text { contabilidad, es decir } \\
\text { logran obtención de } \\
\text { información financiera de } \\
\text { las operaciones realizadas } \\
\text { en un periodo (Elizalde, } \\
\text { 2019). }\end{array}$} & \multirow{14}{*}{$\begin{array}{l}\text { La variable fue evaluada a } \\
\text { través de una guía } \\
\text { entrevista y ficha de } \\
\text { registro creada a partir de } \\
\text { las dimensiones } \\
\text { establecidas por Elizalde } \\
\text { (2019), las misma que fue } \\
\text { dirigido al personal y } \\
\text { aplicada a documentación } \\
\text { del área contable. }\end{array}$} & \multirow{3}{*}{ Sistematización } & Diseño de los procedimientos & \multirow{13}{*}{ Nominal } \\
\hline & & & & Adaptabilidad con el sistema & \\
\hline & & & & Manual de sistematización & \\
\hline & & & \multirow{4}{*}{ Procesamiento } & $\begin{array}{llll}\text { Captación } & \text { de } & \text { los } & \text { datos } \\
\text { financieros } & & & \end{array}$ & \\
\hline & & & & $\begin{array}{l}\text { Clasificación de los datos } \\
\text { financieros }\end{array}$ & \\
\hline & & & & Registro de datos financieros & \\
\hline & & & & $\begin{array}{l}\text { Elaboración de estados } \\
\text { financieros }\end{array}$ & \\
\hline & & & \multirow{3}{*}{ Evaluación } & $\begin{array}{l}\text { Análisis de la información } \\
\text { financiera }\end{array}$ & \\
\hline & & & & Aplicación de métodos & \\
\hline & & & & $\begin{array}{l}\text { Designación } \\
\text { responsabilidades }\end{array}$ & \\
\hline & & & \multirow{3}{*}{ Información } & Presentación del informe & \\
\hline & & & & Contenido del informe & \\
\hline & & & & Oportunidad de la información & \\
\hline & & & Inversión & Costo Instalación & Razón \\
\hline
\end{tabular}


Tabla 2

Proceso contable

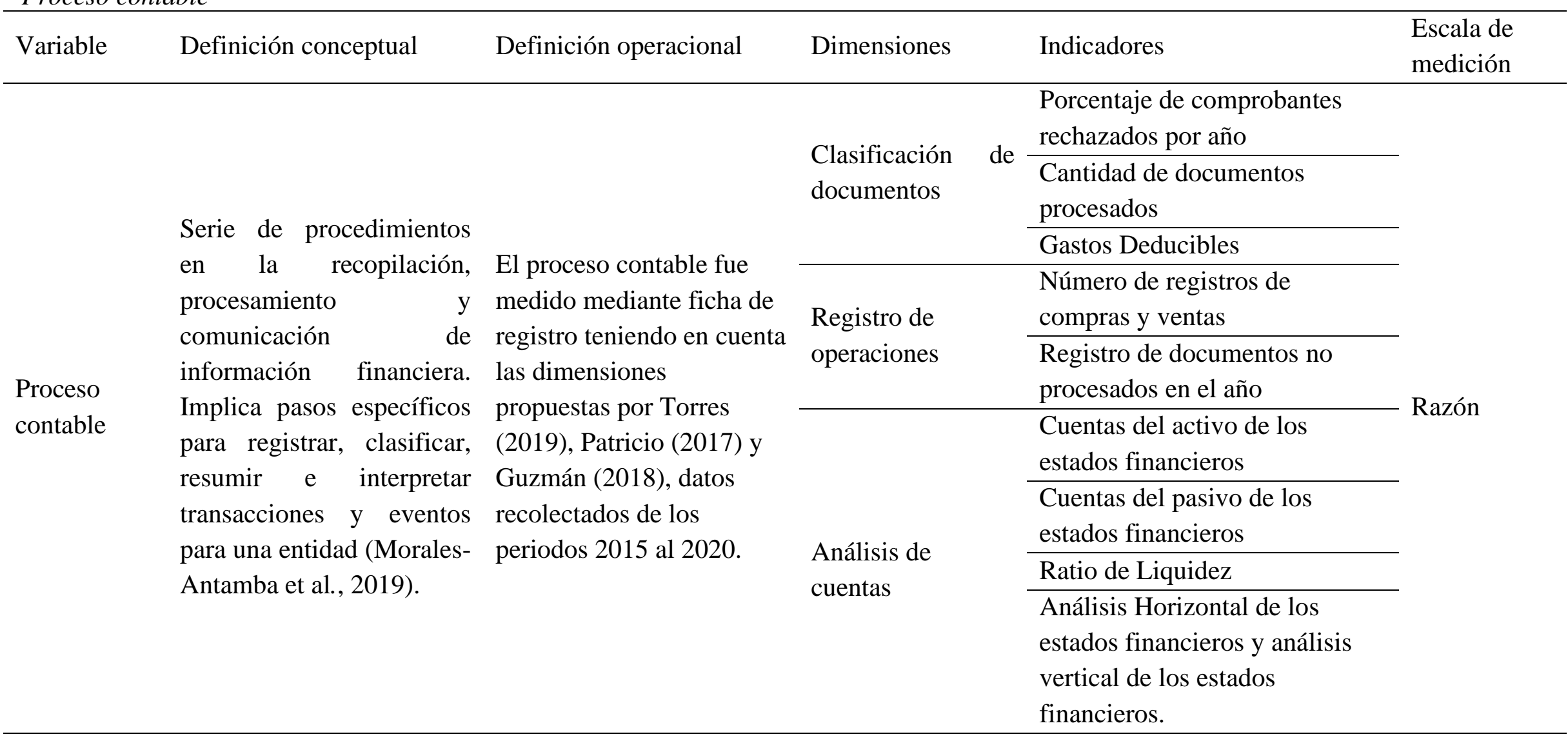




\subsection{Población, muestra y muestreo}

Población: Empresa del rubro Agroindustrial

Muestra: Una empresa del rubro Agroindustrial en los periodos 2015 y 2020.

Muestreo: Por conveniencia

\subsection{Técnica e instrumentos}

\section{Técnica}

Para la variable uso de sistemas de información, se consideró la entrevista, que fue aplicada al personal del área contable y el análisis documental fue aplicada a documentación del área contable de una empresa Agroindustrial del distrito de San Hilarión.

Para la variable proceso Contable, se consideró el análisis documental y fue aplicada a documentación al área contable de una empresa Agroindustrial del distrito de San Hilarión.

\section{Instrumentos de recolección de datos}

El instrumento de recolección de datos aplicados a la variable uso de sistemas de información, fue la guía de entrevista, ficha de registro y para la variable proceso contable se utilizó la ficha de registro.

\subsection{Procedimientos}

Se coordinó con el Gerente General de la empresa para poder obtener información y acceder al personal. Además, se envió la entrevista al correo electrónico de cada personal del área contable, dándoles indicaciones para el llenado, asimismo se contactó al contador de la empresa para que nos facilite información de la documentación, para realizar el análisis documental y poder identificar el estado y la evaluación de etapas del proceso contable en la empresa.

\subsection{Método de análisis de datos}

En la investigación se realizó el análisis descriptivo, la variable uso de sistemas de información se utilizó la guía de entrevista y ficha de registro, para conocer el comportamiento de la variable proceso contable se utilizó la ficha de registro. Para determinar el efecto de la variable uso de sistema de información en la variable proceso contable se consideró evaluar las etapas del proceso contable comprendida los periodos 2015 y 2020.

\section{RESULTADOS Y DISCUSIÓN}

Analizar el uso de un sistema de información dentro de la empresa agroindustrial del distrito de San Hilarión.

\section{Tabla 3}

Analizar el uso de un sistema de información

\begin{tabular}{lll}
\hline \multicolumn{2}{c}{ Indicadores } & \multicolumn{1}{c}{ Acciones de la empresa } \\
\hline Diseño de los procedimientos & $\begin{array}{l}\text { Establecer procedimientos que ayuden a tener información } \\
\text { resumida y precisa }\end{array}$ \\
\hline Adaptabilidad con el sistema & Herramienta que facilita los registros de información \\
\hline Manual de sistematización & Ayuda a llevar el manejo correcto y claro \\
\hline $\begin{array}{l}\text { Captación de los datos } \\
\text { financieros }\end{array}$ & $\begin{array}{l}\text { Se utilizan para respaldar todas y cada una de las } \\
\text { transacciones }\end{array}$ \\
\hline Clasificación de los datos & Conforme se registre cada uno de las operaciones \\
\hline
\end{tabular}


financieros

Registro de datos financieros Registro habitual de las operaciones financieros

Elaboración de estados

financieros

Con la intención de tener información oportuna

Análisis de la información

financiera

Aplicación de métodos

Conocer el estado de la empresa.

Designación

responsabilidades

Aplica métodos a fecha fija y métodos de análisis históricos.

Presentación del informe

de Cumplir con las obligaciones fiscales a tiempo

Contenido del informe

Ayude a ver la realidad de la empresa.

Proyectan información de los estados financieros en forma resumida.

Oportunidad de la información

Evitar multas y sanciones tributarias por incumplimiento de pago.

Nota: Con la aplicación de la entrevista al contador y a sus asistentes, de la empresa en estudio dio como resultado que el uso de sistema de información tiene coincidencia con los resultados de la empresa, puesto que facilita desarrollar sus diversas actividades, por ende, es la columna estratégica de información que facilita tener informes oportunos, para la toma de decisiones.

\section{Tabla 4}

Costo de instalación y gasto de mantenimiento.

\begin{tabular}{lcccccc}
\hline \multicolumn{1}{c}{ Indicadores } & $\mathbf{2 0 2 0}$ & $\mathbf{2 0 1 9}$ & $\mathbf{2 0 1 8}$ & $\mathbf{2 0 1 7}$ & $\mathbf{2 0 1 6}$ & $\mathbf{2 0 1 5}$ \\
\hline Costo de instalación & 0.00 & 0.00 & 0.00 & 0.00 & $50,000.00$ & 0.00 \\
\hline $\begin{array}{l}\text { Gasto de } \\
\text { mantenimiento }\end{array}$ & $1,084.80$ & 994.20 & $1,011.90$ & 972.00 & 0.00 & 0.00 \\
\hline
\end{tabular}

Nota: En la tabla, se aprecia que la empresa en el 2015 no contaba con un sistema de información que le ayude a llevar el orden de sus transacciones económicas y financieras; en el 2016 opta por implementar un sistema de información, como herramienta de ayuda, para tomar decisiones. La implementación tuvo un costo inicial de S/. 50,000.00, lo que incurre de manera adicional y de forma anual a un gasto de $\$ 300.00$ por el concepto de mantenimiento, estos montos se verán variados en soles de acuerdo al tipo de cambio de cada año, se puede apreciar una diferencia mínima en soles entre los periodos 2017 al 2020, el monto de S/. 112.80.

Identificar el estado del proceso contable dentro de una empresa agroindustrial del distrito de San Hilarión.

\section{Tabla 5}

Porcentaje de comprobantes rechazados

\begin{tabular}{ccccccc}
\hline Indicador & 2020 & 2019 & 2018 & 2017 & 2016 & 2015 \\
\hline Porcentaje de comprobantes rechazados por año & $1.2 \%$ & $1.4 \%$ & $1.1 \%$ & $1.1 \%$ & $1.5 \%$ & $4 \%$ \\
\hline
\end{tabular}

Nota: Según los informes revisados, de manera mensual la empresa lleva un control de los comprobantes rechazados por presentar borrones, manchas y enmendaduras. El porcentaje de comprobantes rechazados de los periodos objetos de estudio, se puede apreciar en el periodo 2015 sin el uso del sistema de información un porcentaje de $4 \%$ de comprobantes rechazados, en el periodo 2016 el 1.5\%, con el uso del sistema de información se aprecia una disminución de $2.5 \%$, en comparación con el periodo 2015, paulatinamente en los periodos 2017 y 2018 refleja aún más la 
disminución en 2.9\%, en comparación con el periodo 2015; en los periodos 2019 y 2020, refleja una variación de $2.6 \%$ y $2.8 \%$ en comparación con el periodo 2015.

\section{Tabla 6}

Documentos no procesados

\begin{tabular}{ccccccc}
\hline Indicador & 2020 & 2019 & 2018 & 2017 & 2016 & 2015 \\
\hline Documentos no procesados en el año & 0 & 0 & 0 & 1 & 3 & 15 \\
\hline
\end{tabular}

Nota: Se puede apreciar el registro de aquellos comprobantes que no fueron registrados en el periodo que corresponden, según el registro de compras física del periodo 2015 donde la empresa no hacía uso de un sistema de información, se registró 15 comprobantes de diciembre 2014, con el uso del sistema de información se aprecia que en el periodo 2016 se registró 3 comprobantes de diciembre del 2015, con una diferencia de 12 comprobantes menos que en el periodo 2015, en el periodo 2017 solo se registró 1 comprobante de diciembre del 2016; con una diferencia de 14 comprobantes menos a comparación con el periodo 2015; en los periodos respectivos 2018, 2019 y 2020 no se registró documentos no procesados. 
Tabla 7

\section{Estado de resultados}

\begin{tabular}{|c|c|c|c|c|c|c|}
\hline & 2020 & 2019 & 2018 & 2017 & 2016 & 2015 \\
\hline Ventas & $1,460,087$ & $1,068,806$ & 671,067 & $1,651,065$ & $1,108,513$ & 613,552 \\
\hline (-) Descuentos y bonificaciones & 0 & 0 & 0 & 0 & 0 & 0 \\
\hline (=) Ingresos operativos netos & $1,460,087$ & $1,068,806$ & 671,067 & $1,651,065$ & $1,108,513$ & 613,552 \\
\hline (-) Costo de Ventas & 0 & 0 & 0 & 0 & 0 & 0 \\
\hline (=) Resultado bruto & $1,460,087$ & $1,068,806$ & 671,067 & $1,651,065$ & $1,108,513$ & 613,552 \\
\hline \multirow[t]{2}{*}{ (-) Gastos Administrativos } & $-848,241$ & $-526,940$ & - & $-846,547$ & $-534,727$ & . \\
\hline & & & 345,712 & & & 342,007 \\
\hline \multirow[t]{2}{*}{ (-) Gastos de Ventas } & $-468,442$ & $-447,833$ & - & $-701,899$ & $-498,666$ & - \\
\hline & & & 265,204 & & & 228,005 \\
\hline $\begin{array}{l}\text { (=) Resultado de las } \\
\text { operaciones ordinarias }\end{array}$ & 143,405 & 94,033 & 60,150 & 102,618 & 75,120 & 43,539 \\
\hline (+) Ingresos financieros & 0 & 0 & 0 & 3 & 0 & 0 \\
\hline (-) Gastos financieros & 0 & 0 & 0 & -27 & $-19,628$ & 0 \\
\hline (+) Ingresos extraordinarios & 0 & 0 & 0 & 0 & 18,000 & 0 \\
\hline (-) Gastos extraordinarios & 0 & 0 & 0 & 0 & 0 & 0 \\
\hline $\begin{array}{l}\text { (+) Ingresos de ejercicios } \\
\text { anteriores }\end{array}$ & 0 & 0 & 0 & 0 & 0 & 0 \\
\hline $\begin{array}{l}\text { (-) Gastos de ingresos } \\
\text { anteriores }\end{array}$ & 0 & 0 & 0 & 0 & 0 & 0 \\
\hline $\begin{array}{l}\text { (=) Resultado ANTES del } \\
\text { impuesto }\end{array}$ & 143,405 & 94,033 & 60,150 & 102,594 & 73,492 & 43,539 \\
\hline (-) Impuesto a la renta & $-43,021$ & $-28,210$ & $-18,045$ & 30,778 & $-22,048$ & $-13,062$ \\
\hline (=) Resultado neto & 100,383 & 65,823 & 42,105 & 133,372 & 51,444 & 30,477 \\
\hline
\end{tabular}

Nota: en el estado de resultado de ven reflejados de manera resumida los movimientos de los ingresos y gastos de la empresa.

Determinar el efecto del uso de un sistema de información en el proceso contable de una empresa agroindustrial

\section{Análisis:}

En el periodo 2015, la empresa venía desarrollando sus actividades de manera tradicional, lo que lo dificultaba atrasaba con la presentación de sus estados financieros conforme al cronograma, establecido por SUNAT; en los periodos 2016 al 2020 con el uso del sistema de información, la empresa mejoró en el desempeño de sus procesos, y facilito tener información resumida, presentando sus estados financieros de manera oportuna.

En la presente investigación como primer objetivo específico se planteó analizar el uso de un sistema de información dentro de una empresa agroindustrial. Según Elizalde (2019) El uso del sistema de información está comprendida por la sistematización, procesamiento, evaluación, información e inversión para ser más eficientes y tienen el objetivo de llevar la contabilidad, es decir logran obtención de información financiera de las operaciones realizadas en un periodo. Asimismo, Macías et al. (2020), precisaron que el uso de los sistemas de información en el área contable facilita el cambio de datos con mayor facilidad, fiabilidad y rapidez, ya que reúne información útil y excluye operaciones innecesarias para la toma de decisiones en las organizaciones. En los resultados obtenidos del presente estudio se encontró que con el uso de un sistema de información la empresa mejoró en el desempeño de sus procesos al tener información 
resumida y oportuna, siendo esta información vital para la toma de decisiones dentro de la empresa. Los resultados coinciden con los aportes de Andarwati et al. (2020), quienes indican que el sistema de información desempeña un papel importante dentro las Pymes; muestra la facilidad de uso, la calidad del sistema de información y la satisfacción del usuario final de la información contable. Estos resultados evidencian que la implementación de un sistema de información, ayudó a mejorar positivamente en el desempeño del proceso contable de la empresa, son consideradas por los autores de los antecedentes, Según los aportes de Agustín et al. (2020), sostienen que el uso del sistema de información genera información de calidad y apoya en las actividades internas de la empresa.

Como segundo objetivo específico se planteó identificar el estado del proceso contable dentro de una empresa agroindustrial, según Morales-Antamba et al. (2019), el proceso contable rige una serie de procedimientos en la recopilación, procesamiento y comunicación de información financiera, asimismo, implica pasos específicos para registrar, clasificar, resumir e interpretar transacciones y eventos para una entidad; según Rodríguez et al. (2019) definen que los procesos contables están ayudados por el uso de sistema de información y la dirección que deben obtener las entidades para la utilización de los mismo; además, las causas que influyen a los procesos contables a la toma de decisiones mediante la ayuda especializada (Moreira et al., 2019). En los resultados encontrados de la presente investigación se evidencia que el proceso contable de la empresa mostró mejoras con el uso del sistema de información; con la reducción de errores en la presentación de sus informes financieros, convirtiéndose en un soporte primordial para encaminar a la empresa y tomar decisiones. El resultado de la presente investigación coincide con los aportes de Rodríguez et al. (2019) quienes consideran que el proceso contable es una herramienta financiera que se enfoca como fórmula para el desarrollo de las microempresas. Estos resultados evidencian que el proceso contable mejoró positivamente con la implementación del sistema de información; son consideradas por autores, según los aportes de Valdez et al. (2019), quienes se refieren que los procesos contables dentro de una organización expresan las políticas, procedimientos, controles; disminuyendo los desaciertos y presentando razonabilidad a los estados financieros.

Como objetivo general se planteó determinar el efecto del uso de un sistema de información en el proceso contable de una empresa agroindustrial, según los aportes de Farida et al. (2021) señalaron que, el uso del sistema información sirve para la producción de información sobre la base de la cual se toman las decisiones, ya que juega un papel importante y destacado dentro de las organizaciones. Por su parte, Nata y Sari (2021) sostuvieron que el uso de sistemas de información ha hecho posible llevar la contabilidad a un nuevo nivel, ya que con el uso eficiente ayuda a las empresas almacenar y recopilar todos los movimientos y estar actualizados. En los resultados encontrados de la presente investigación el uso de un sistema de información tiene un efecto positivo en los procesos contables dentro de la empresa agroindustrial del distrito de San Hilarión, y eso se ve reflejado en los estados financieros, con los totales del activo, pasivo más patrimonio, en S/. 1,699,733 sin el uso del sistema de información en el periodo 2015, y con el uso del sistema de información de S/. 1,930,216 para el periodo 2016, de S/. 1,948,049 para el periodo 2017, de S/. 1,963,861 para el periodo 2018, de S/. 2,018,415 para el periodo 2019 y de S/. 2,245,874 para el periodo 2020, evidenciando que el uso del sistema de información influirá y tendrá un impacto de mejora que permitirá lograr el objetivo de contar con información oportuna, convirtiéndose un pilar estratégico en el proceso contable de la empresa.

El resultado de la presente investigación se asemeja con los aportes de Pervan y Dropulic' (2019), quienes indican que el efecto de uso de un sistema de información provoca cambios completos y significativos en la contabilidad de la empresa, reduce el tiempo de presentación de informes, aumenta la frecuencia de presentación de informes; el efecto del uso de sistema de información permite una producción más rápida, y brinda la oportunidad de crear nuevos informes.

Los antecedentes utilizados muestran que lo evidenciado en la presente investigación sobre el efecto del uso de un sistema de información es relevante en las etapas del proceso contable, puesto que esto facilitaría tener información útil para la toma de decisiones en la empresa, según Andarwati et al. (2020) el sistema de información proyecta información en forma resumida y de fácil análisis. 


\section{CONCLUSIÓN}

De acuerdo a los resultados, se llegaron a las siguientes conclusiones:

El uso de un sistema de información genera un efecto positivo en el proceso contable de una empresa agroindustrial del distrito de San Hilarión, al realizar el análisis, en el 2015 la empresa llevaba el control sus operaciones en forma manual, y al no usar un sistema de información, estaba expuesta a la acumulación de errores, y a hacerse acreedor de alguna multa o sanción, en 2016 con el uso de un sistema de información se encontró mejoras, en el registro de las transacciones, reduciendo el tiempo de presentación de los estados financieros, ayudando a ver en forma resumida la realidad de la empresa.

Al analizar el uso de sistema de información en la empresa agroindustrial del distrito de San Hilarión, se ha ido viendo paulatinamente el respaldo de sus registros y control de sus documentaciones, con la disminución periodo tras periodo del porcentaje de comprobantes rechazados, y de aquellos comprobantes que no hayan sido registrados en el periodo que corresponden, creando seguridad de cada uno de sus movimientos, asimismo a los directivos de la empresa el uso de un sistema de información les facilita tener de manera resumida y oportuna información útil para tomar decisiones, también excluye las operaciones innecesarias y ayuda en las diligencias internas de la empresa.

Se concluye que el proceso contable dentro de la empresa agroindustrial del distrito de San Hilarión, parte con la clasificación de documentos, registro de operaciones y análisis de cuentas, estos se expresan en los estados financieros donde se puede ver en forma resumida sus gastos e ingresos, para así realizar proyecciones y se eviten crisis que puedan llevar a la empresa a un estancamiento.

\section{REFERENCIAS}

Agustin, R. D., Firdaus, M., \& Widaninggar, N. (2020). Determinants of Accounting Information System in pt. Indomarco Adi Prima, Jember Branch. International Journal of Environmental, Sustainability, and Social Sciences, 1(1), 70-77. https://doi.org/10.38142/ijesss.v1i1.19

Andarwati, M., Zuhroh, D., \& Amrullah, F. (2020). Determinants of Perceived Usefulness and End-User Accounting Information System in SMEs. International Journal of Advanced Science and Technology, 29(8), 46-61. Obtenido de http://sersc.org/journals/index.php/IJAST/article/view/10415/5609

Castillo, G., \& Pérez, E. (2017). Diagnóstico de los sistemas de información en las empresas priorizadas según los requerimientos actuales. Palabra clave (La plata), 6(2), 1-11. https://doi.org/10.24215/PCe022

Crespo, M. K., Carchi, K. L., Zambrano, Á. A., Orellana, D. A., \& Gonzalez, S. E. (2020). Mejora Continua en el proceso contable y su aporte en la competitividad de las MIPYMES en la Provincia de El Oro (Ecuador). Revista Espacios, 41(1), 3. Obtenido de http://www.revistaespacios.com/a20v41n01/a20v41n01p03.pdf

Elizalde, L. (2019). Gestión contable como base fundamental para las decisiones generales. Revista de contabilidad y auditoría, 32-44. Obtenido de http://cimogsys.espoch.edu.ec/fade/public/docs/contauditar/Articulo3.pdf

Farida, I., Mulyani, S., Akbar, B., \& Setyaningsih, S. (2021). Quality and efficiency of accounting information systems. Utopía y Praxis Latinoamericana, 26(2), 323-336. https://doi.org/10.5281/zenodo.4678910

Fuentes-Doria, D. D., Toscano-Hernández, A. E., Díaz-Ballesteros, J. L., \& Escudero-Garrido, Y. (2019). Ética y sostenibilidad en la gestión contable: Un análisis documental en el contexto Mundial en los periodos 20092018. Contaduría Universidad De Antioquia (75), 43-67. https://doi.org/10.17533/udea.rc.n75a02

Guzmán, M. d. (2018). Toma de decisiones en la gestión financiera para el sistema empresarial (Primera ed.). Guayaquil, Ecuador: Editorial Grupo Compás. Obtenido de http://142.93.18.15:8080/jspui/bitstream/123456789/278/1/LIBRO\%20LISTO-ilovepdf-compressed-2.pdf

Macías-Collahuazo, E. X., Esparza-Parra, J. F., \& Villacis-Uvidia, C. A. (2020). Las tecnologías de la información y la comunicación (TICs) en la contabilidad 
Morales-Antamba, L. R., Sánchez-Cando, A. M., Viscaino-de la Cruz, C. J., \& Avellán-herrera, N. A. (2019). Importancia de los fundamentos contables. Aplicación práctica de un proceso contable en una empresa comercial. Revista de Investigación Sigma, 6(1), 84-100. http://dx.doi.org/10.24133/sigma.v6i01.1233

Moreira, L., García, N., Granda, M., Samaniego, H., \& Lema, M. D. (2019). Impacto de la aplicación de un proceso contable y gestión de inventarios para la compañía DETEICELI. Revista Dilemas Contemporáneos: Educación, Política y $\quad$ Valores., $3(92), \quad 1-16 . \quad$ Obtenido de https://www.dilemascontemporaneoseducacionpoliticayvalores.com/index.php/dilemas/article/view/1830

Nata, P., \& Sari, N. L. (2021). The effect of the effectiveness of accounting information systems, implementation of good governance, and organizational culture on financial performance. American Journal of Humanities and Social Sciences Research, 5(7), 84-92. Obtenido de https://www.ajhssr.com/wpcontent/uploads/2021/07/K21578492.pdf

Patricio, C. (2017). Manual de usuario del sistema de información de gestión económica y financiera. Lima: Real Systems S.A. Obtenido http://unijuanpablo.edu.pe/manuales/GESTION_ECONOMICA_FINANCIERA.pdf

Pervan, I., \& Dropulic', I. (2019). The impact of integrated information systems on management accounting: Case of Croatia. Journal of contemporary management issues, 24(1), 21-38. https://doi.org/10.30924/mjcmi.24.1.2

Rodríguez, A., Velasteguí, L., Maldonado, H., Benalcázar, M., \& Benalcázar, Y. (2019). Proceso contable: herramienta financiera para la toma de decisiones en las PYMES Parroquia El Esfuerzo. Revista Dilemas Contemporáneos, $\quad$ VI (42), 1-22. $\quad$ Obtenido https://www.dilemascontemporaneoseducacionpoliticayvalores.com/index.php/dilemas/article/view/1397

Torres, A. (2019). Elementos de un sistema de información contable efectivo. Quipukamayoc, $27(53), 73-79$. http://dx.doi.org/10.15381/quipu. v27i53.15988

Valdez, W. P., Narváez, C. I., Ormaza, J. E., \& Erazo, J. C. (2019). Procedimientos contables y su incidencia en la sistematización de la información de la Cooperativa de Ahorro y Crédito Atlántida de la ciudad de Cañar. Visionario Digital, 3(2.1.),497-526. https://doi.org/10.33262/visionariodigital.v3i2.1.586 